Four weeks after the operation, the wound being completely cicatrised, she was allowed to move about in her room; and, just six weeks from the date of the ligature of the artery, she descended three long fights of stairs, walked for some time in the strcets, and reascended the steps to her lodgings; and, though fatigucd by the effort, she felt next day all the better for it. On March 3ist, she called to see me, much improved in strength, though with still some tendency to swelling of the legs, especially the left, when in the erect posture. The aneurismal swelling felt merely like a slight glandular thickening. [On July 25th, 1868, ] again visited her. She had derived much benefit from a stay at the seaside, and the tendency to odema of the extremities was greatly diminished. There was still absence of pulsation in the external iliac artery, and the cicatrix remained quite sound twenty-five weeks after the opera. tion; so that, whatever may have become of the ligature, there need be no apprehension of its ever making its appearance externally.]

The success of these cases of ligature depends, as we have seen, upon the circumstance that not only a neutral foreign body, but a portion of dead tissue, if simply protected from putrefaction, is entirely devoid of irritating properties. A good example of this fact is presented by a case at present under my care.

Case of Acute Necrosis treated on the Antiseptic System.-The patient is a boy, eight years of age, who was admitted into the Infirmary on Jan. 25th, 1868, having, five days previously, received a violent blow upon the left leg with a heavy pair of tongs, resulting in intense inflammation in the limb, which was red and swollen from the knee to the ankle. Fluctuation being distinctly perceptible over the upper part of the tibia, the matter was evacuated antiseptically. A piece of lint dipped in an oily solution of carbolic acid having been laid upon the part where the incision was to be made, its lower edge was raised to allow a knife smeared with the same solution to be plunged into the cavity of the abscess, when the curtain was at once dropped so that the pus might flow out beneath it. When all the matter had been pressed out, an external antiseptic dressing was applied, and this was afterwards changed daily. Four other abscesses afterwards made their appearance at intervals down the limb. These were treated in the same way; and in every case when a probe, carefully guarded by being passed among folds of lint steeped in the antiseptic oil, was introduced into the incision, it came into contact with bare bone. This was of itself sufficient evidence that portions of the tibia, of greater or less thickness, were dead; for, had the periosteum been raised by suppuration from living bone, the osseous surface would have become covered with granulations during the process. But evidence which must satisfy the most incredulous is af forded by the fact that, between two and three weeks after the first abscess was opened, a probe introduced into the orifice still passed down to bare bone. Under such circumstances, what, it may be asked, could be the advantage of continuing the antiseptic dressing? If dead bone was present, whether in larger or smaller amount, must it not become detached from the living osseous tissue by a gradual process of exfoliation, which an antiseptic applied to the skin could neither promote nor hinder? Such may be a natural inquiry. But having seen a large mass of dead bone absorbed before my eyes by the granulations that enveloped it, in a case of compound fracture treated antiseptically (see Lancet, March 23rd, 1867); and having also had evidence from post mortem examination in a case of hip-joint disease where extensive necrosis existed in connection with caries, that bone killed by inflamma. tion might, under antiseptic management, fail to induce suppuration; putting those facts together, I thought it not unlikely that, in the case we are considering, the dead portions of the tibia would be absorbed by the living tissues around them, if we persevcringly maintained an effectual external antiseptic guard. Such, then, was the practice we pursued, and the result was such as $I$ had anticipated. The various incisions successively healed, till by the 6th of this month (April), eleven weeks after the receipt of the injury which caused the attack, the abscess last opened was soundly closed and cicatrised, not a particle of dead bone having come away from any of the openings. At the same time, the swelling of the limb, instead of increasing, as is the case under ordinary treatment, from formation of new bone in the periosteum under the stimulating influence of exfoliations soaked with putrid liquids, had disappeared almost entirely from the upper part of the leg, which was that primarily affected, and was rapidly diminishing elsewhere. [On the 18th of May, the boy left the hospital with the full use of the limb. I had intended giving him a somewhat longer rest in bed as a measure of precaution. But I found that for a considerable time it had been impossible to keep him from getting up and running about the ward; and he was none the worse for his activity.] This certainly was very different from the tedious course of such cases under ordinary treatment.

With regard to the manner in which the dead bone has been disposed of, some who have not witnessed similar occurrences may doubt the possibility of its absorption, and believe that the necrosed pieces are still lying unchanged in the interior of that leg. But even those who take such a view must admit that we have here a most striking illustration of the important truth, that dead tissue, if protected from putrefaction, is of itself incapable of exerting any disturbing influence upon surrounding parts.

(To be concluded.)

\section{THE PATHOLOGY AND TREATMENT OF SUN-STROKE.}

BY GEORGE JOHNSON, M.D., F.R.C.P., Professor of Medicine in King's College; Physician to King's College Hospital.

Sitn-Stroke or Heat-Apoplexy, more corrcctly' Heat-Apnoea.-Causes.Symptowes. - Morbid Anatomy. - Pathology. - The over-heated Blood reiaxes the Mintute Pulmonan' Arteries, and so floods the Capillaries. - The Slate of the Lungs in Cholera contrasted with that in Heat. Apnaa.-Treatment.- The neain object is to cool the over-heated Blood, so as to restore the Contractile Power of the Minute Pulmonary Arteries, to lessen the Capillary Engorgement of the Lungs, and thus temove the State of Apnoea.

THE formidable disease known by the name of sun-stroke or heatapoflexy might be more correctly designated heat-apnaca, as I shall presently endeavour to prove. Although this affection frequently occurs from direct exposure to the sun's rays, it is also of common occurrence without such exposure. The one essential and constant condition is a very high temperature of the air. The most powerful concurring causes are, muscular exertion and excessive fatigue; hot clothing, and especially such as tends to impede the respiratory movements; an excessive use of alcoholic liquors; and the close and impure air of hot and overcrowded rooms. The disease may be fatal in a few minutes, or the symptoms may last from one to forty-eight hours.

The rapidly fatal cases are spoken of as belonging to the cardiac variety. The patient falls unconscious, gasps, and dies. When the disease runs a less rapid course, it is said to be of the cerebro-spitzal variety. There are great heat, dryness, and redness of the skin, giddiness, nausea, congestion of the eyes, and frequent desire to micturate ; sometimes delirium, then drowsiness, passing into coma. The pupils are contracted ; the breathing is hurried and laborious; the heart's action is tumultuous; the pulse rapid, at first distinct, but soon becoming feeble and irregular. Convulsions are of common occurrence, either early in the attack, or immediately before death. After death, however rapid may have been the course of the disease, the one constant condition is extreme, "unexampled" congestion of the lungs, with distension of the right side of the heart.

Dr. Maclean, to whose article on Sun-Stroke (Reynolds's System of Medicine) I would refer for a clear and succinct account of the facts of the disease, states that all modern pathologists are agreed that the superheating of the blood, which precedes and accompanies sun-stroke, has a depressing, and not a stimulating, effect on the nervous centres. In what way, then, does the over-heated blood exert this depressing effect on the nervous centres? I will endeavour to give what I believe to be the true physiological explanation of the phenomena.

The hot blood relaxes the muscular walls of the minute pulmonary arteries. The pulmonary capillarics are consequently flooded with blood. This overfulness of the capillaries interferes with the aëration of the blood. In fact, the overgorged vessels must encroach upon the pulmonary vesicles, and so diminish the air-space within the lungs: while the air itself is highly rarefied. Hence a state of more or less complete apnœe. Unaërated blood is sent to the muscular tissue of the heart, and to the brain : hence the cardiac and the cerebral symptoms. A similarly engorged statc of the cutaneous capillaries, consequent upon extreme relaxation of the minute arteries, is the probable cause of the dryness of the skin. An excessively engorged state of the capillaries is as unfavourable for cutaneous secretion as it is for pulmonary respiration. The dry and inactive state of the skin and the want of surfaceevaporation tend to elevate still more the temperature of the blood; and the suppressed cutaneous secretion, being diverted to the kidneys, probably alters the quality of the urine, renders it irritating to the bladder, and explains the frequent micturition.

This explanation of the phenomena is confirmed by the results of treatment. There is now a very general concurrence of opinion that the application of cold to the skin is the most successful remedy. The object to be kept in view is not merely, as it is generally stated, to cool the skin, or to excite the respiratory movements by the stimulus of the 
dowehe, but to cool the blood; and thus to restore the contractility of the minute arteries of the langs. The condition of the pulmonary vessels in this disease is the exact opposite to their state in cholera collapse. In cholera collapse, the minute pulmonary arteries are in a state of extreme contraction; and, as a consequence, the capillaries are extremely anzemic. In heat-apnoea, the pulmonary arteries are extremely relaxed; and the capillaries, consequently, are excessively engorged. In cholera collapse, external warmth in some degree, but much more rapidly and decidedly a warm injection into the veins, relaxes the arterial spasm, and restores the circulation. In heat-apncea, on the contrary, the object is to cool down the over-heated blood, so to revive the contractile power of the minute pulmonary arteries, to relieve the capillaries from their embarrassing excess of blood, and thus to remove the state of apnoea. A clear apprehension of these physiological principles cannot fail to be of great assistance in practice.

In the treatment of heat-apnoea, the following appear to be the main points which require attention. The patient should be placed in a recumbent position in the coolest possible place, with a free current of air. The clothes should be removed, and cold water applied to the whole surface; or, if the symptoms be urgent, the clothes should immediately be saturated with cold water without waiting to remove them. If the respiratory movements be failing and feeble, the cold douche is a powerful excitor; but if the breathing be rapid and laborious, it is better to envelope the body in a wet sheet, and to quicken evaporation and cool. ing by a fan or a pair of bellows. If the patient can swallow, let him drink iced water freely. Whether he can swallow or not, iced water may from time to time be injected. The marvellous effect of hot venous injections in cholera collapse, and the urgent need for cooling the blood in heat-apncea, suggest the expediency, in extreme cases, of injecting into a vein the same saline solution as has so frequently been employed in cholera, only injecting it cold instead of hot.

Dr. Morehead (Clinical Researches on Diseases in India) recommends venesection in the early stages of the attack, while Dr. Maclean condemns the practice. A routine practice of venesection would be destructive; but, when symptoms of excessive venous engorgement are present, 2 cautious venesection would be quite justifiable, and probably beneficial, on the well known principle of lessening distension of the right side of the heart, and thus increasing its contractile power. When respiration has suddenly and quite recently ceased, artificial respiration by Dr. Silvester's method may possibly restore animation. While symptoms of apnoes continue, however great may be the apparent exhaustion, no alcoholic stimulants are to be given, for the reason that alcohol, as well as anæsthetic vapours and narcotics, impede oxidation of the nervous and other tissues, and therefore increase the risk of death from apnoea. Ammonia may be applied to the nostrils as a stimulant, and, if the pa. tient can swallow, it may be given internally. Ammonia is a powerful diaphoretic, and the restoration of the cutaneous secretion is an important step towards recovery. When the skin becomes cool and moist, of course all cold applications are to be discontinued. To sum up thenas hot air and hot blood are the cause of this form of apnoea, so cold air and cold water are the chief means of cure; all other means are subsidiary to these.

\section{NOTES ON THE SURGERY OF THE NINETEENTH CENTURY AND THE OPHTHALMOSCOPE.*}

BY JAMES VOSE SOLOMON, F.R.C.S., Surgeon to the Birmingham and Midland Eye Hospital, etc.

IN the very eloquent and learned address on Surgery, delivered at the Dublin meeting of the British Medical Association by Professor Smith, it was deplored that so little attention is paid, in the present day, to the Patristic literature of our profession; and it was asserted that in those noble writings were to be found some things which had in our own day been presented as new discoveries.

Assenting then, as 1 do still, to much contended for by Professor Smith, a man of great modesty and of rare gifts and acquirements, I yet felt at the time of the delivery of his address, and on reflection find no reason to alter my opinion that the nineteenth century surgeon has no occasion for shame at the present state of his art. Can he not fairly boast that in his day scalpel and microscopic human anatomy attained perfection; that the true nature of the inflammatory process was understood, and a rational and successful treatment adopted for its relief? He can

* Read before the Birmingham and Midland Coanties Branch. claim that in his day the knife was robbed of its terrors by chloroform and local ansesthetics; while Eve's curse was mitigated by the same divine agency. The surgery of tendons, that of the iris, and that of acute glaucoma, were perfected.

I believe the future historian of the epoch will recognise as character. istic of it a marvellous improvement in the diagnostics of disease, and a large diminution of death-rate; great economy of time in the attainment of a stage of convalescence, with considerable diminution of the restraints and severities incident to our methods of cure; the substitution, for destructive operations, of procedures which reduce the parts submitted to surgical interference as near as is possible to a physiological state; the cure of certain diseases by bloodless operations, where the knife had ever before been the sole remedial resort; the healing of large wounds without occurrence of the process of suppuration. He will dilate with admiring wonder upon the rapid perfection of ovarian surgery, and the accomplishment of this by the labours of scarce more than twelve men-a death-rate of twenty per cent. having been attained. As a striking instance of economy of time, of recovery, and of physiological surgery, he will adduce excision of the elbow-joint in cases of articular disease, whereby years of suffering have been abridged to an easy and almost painless recovery extending over no more than a few weeks. In the list of bloodless operations, we doubt not, will be found the cure of stone in the urinary bladder through its eliminative canal by lithotrity; and, greatest of marvels, the consolidation of aneurism, even of the descending abdominal aorta, by compression. On the other hand, the historian will find much to tell of the invasion, and successful subjuga. tion, by surgery, of districts which had, from the birth of the healing art, been considered as belonging to the domain of pure medicine; as, for example, the successful treatment by operation of acute glaucoma, reflex ophthalmia, recurrent iritis, and, it may be, of myopia. He will attribute a large measure of our progress to the important aids contributed to minuteness and accuracy of diagnosis by physical science, and by mechanical ingenuity and skill. He will relate that the living bladder ceased to be a dark obscure cavity; its diseases being pictured to the surgeon's eye on the field of the endoscope, and encysted and loose calculi being accurately differentiated. He will tell how the pathology of the larynx, painted on the most ancient of mirrors made by the hand of man, came to be unravelled; how the site on the voice-string from which the polypus had been snared was cauterised with unerrring certainty by the electric cautery. He will tell how the pulse wrote its own diary with the sphygmograph. He will dilate on the complete revolution effected in our treatment and views of the nature and pathology of eye-diseases by the discovery and use of the ophthalmoscope. He will, no doubt, attribute the rich harvest of fruit gathered from some of these methods to the enlistment of the sense of sight as an aid to that of touch, or as the sole exponent of the characteristics of the diseased changes-a sense which affords ideas and impressions altogether different from those obtainable by any other means whatever.* While speaking of these triumphs in the art of diagnosis, I cannot here refrain the remark that the tendency of the inventions and methods of the surgery of our period, which has become largely mechanical, is to put more nearly on a level those of gifted intellect and those of moderate understanding. It cannot be doubted, I think, that this levelling process is effecting much good by diffusing the blessings of our art more widely amongst humanity.

Nevertheless, the division of labour, called Specialism, will be maintained, if not increased; and through such division the great body of the profession will, as heretofore, acquire more distinct views of the pathology and appropriate treatment of certain diseases. The public is every day becoming more firmly convinced that their interest lies in supporting this arrangement; and I believe the profession will ere long be quickened to recognise that a cordial co-operation between specialist and general practitioner shuuld become an universal rule, because subserving in the most complete manner the beneficent and benevolent purposes for which the profession exists.

'The death-rate in ovariotomy has been reduced, as I have said, to 20 per cent. In 333 cases of excision of the elbow-joint, 12 per cent. died; 4.2 suffered amputation; in 6.9 per cent. there was ankylosis; and in 256 cases, or 76.87 per cent., there was mobility of the joint. One hundred adult cases of stone in the bladder, occurring consecutively

- Although the touch has been habitually resorted to for ages as a means of diagnosis, the common existence of ulcerative and inflammatory hypertrophy was never even suspected until the speculum revealed their presence. When once, however, the eye has demonstrated the existence of these morbid states, the touch, with its assistance, generally acquires the power of distinguishing the most minute changes; and it then appears quite a marvel to the practitioner that the grossest morbid conditions should previously have always escaped his recognition. In illustration of these remarks, Dr. J. Henry Bennet quotes an instance where an eminent surgcon who had marks, Dr. J. Henry Bennet quotes an instance where an eminent surgeon who had devoted his whole hife to the study of uterine disease, mistook, thus relying on touch
alone, advanced cancerous ulceration of the os uteri for a simple ulcer, and promised that the patient would soon recover. - Infiammation of the Uterws. By J. H. Bennet and edition. Pp. 494-6. 